\title{
Qualitative assessment of pain management in patients undergoing computed tomography-guided transthoracic lung biopsy
}

\author{
Erin T Wong BSc, Carol Dunham RN, Demetris Patsios MD
}

ET Wong, C Dunham, D Patsios. Qualitative assessment of pain management in patients undergoing computed tomography-guided transthoracic lung biopsy. Pain Res Manage 2014;19(3):149-152.

BACKGROUND: Unpublished questionnaire data collected by the authors' institution (Toronto General Hospital, Toronto, Ontario) indicates that patients often experience significant pain while undergoing lung biopsy, yet receive only a fraction of the maximum allowable dosage of analgesic.

OBJECTIVES: To qualitatively assess current pain management practices from the patient perspective. Patient education and patient satisfaction were also evaluated.

METHODS: From March through June 2012, participants were contacted via telephone within one week of their procedure until data saturation was reached. The semistructured interviews were based on a study-specific interview template. Thematic analysis of qualitative study data was used to identify recurring interview topics.

RESULTS: A consecutive sample of 16 outpatients who had undergone image-guided transthoracic lung biopsy at the authors' institution were interviewed. None of the study participants reported noteworthy pain associated with the insertion of lung biopsy needles. The most significant pain was caused by positioning within the computed tomography scanner, particularly among participants who were in the prone position. All participants reported high satisfaction with the amount of analgesic received. Potential complications and recovery period details were identified as areas for improved patient education.

CONCLUSIONS: At the authors' institution, pain associated with lung biopsy needle insertion was well controlled. Positional pain is common for patients required to be in the prone position. Potential solutions include increasing awareness of positional pain and instituting additional supportive equipment.

Key Words: Fine-needle biopsy; Image-guided biopsy; Lung neoplasms; Pain; Solitary pulmonary nodule

U npublished questionnaire data collected by our institution (Toronto General Hospital, Toronto, Ontario) indicates that patients often experience significant pain while undergoing lung biopsy, yet receive only a fraction of the maximum allowable dosage of analgesic. Because anesthesiologists are not usually present (1), radiologists' ability to provide appropriate sedation and analgesia is crucial to minimizing unwanted movement, maintaining hemodynamic stability and improving patient satisfaction (2). The literature also indicates that pain management in the setting of interventional procedures can often be improved (3). To reduce pain during lung biopsy, we interviewed patients to gain a greater understanding of their perception of pain and to identify areas in which additional interventions may be beneficial.

In addition to assessing current pain management practices, the present study evaluated patient education and patient satisfaction. Mueller et al (4) reported that patients with a greater understanding of interventional procedures experience less anxiety. Furthermore, Schupp et al (5) found that high anxiety is a predictor for procedural

\section{L'évaluation qualitative de la gestion de la douleur chez des patients subissant une biopsie transthoracique sous contrôle tomodensitométrique}

HISTORIQUE : D'après les données tirées de questionnaires non publiés colligées par l'établissement des auteurs (Toronto General Hospital, Toronto, Ontario), les patients ressentent souvent de vives douleurs pendant une biopsie pulmonaire, mais ne reçoivent qu'une fraction de la posologie maximale d'analgésique permise.

OBJECTIFS : Procéder à une évaluation qualitative des pratiques de gestion de la douleur selon le point de vue des patients. Évaluer également l'éducation et la satisfaction des patients.

MÉTHODOLOGIE : De mars à juin 2012, on a téléphoné aux participants dans la semaine suivant leur intervention, jusqu'à saturation des données. Les entrevues semi-structurées se fondaient sur un modèle d'entrevue propre à l'étude. L'analyse thématique des données de l'étude a permis de déterminer les sujets d'entrevue récurrents.

RÉSULTATS : On a interviewé un échantillon de 16 patients ambulatoires consécutifs qui ont subi une biopsie transthoracique contrôlée par imagerie à l'établissement des auteurs. Aucun d'eux n'a ressenti de douleur notable à l'insertion de l'aiguille de biopsie pulmonaire. La principale douleur s'associait à la position dans l'appareil de tomodensitométrie, particulièrement chez les participants en décubitus ventral. Tous les participants se sont dits très satisfaits de la quantité d'analgésique administrée. Les patients auraient pu être mieux informés des complications potentielles et de la période de rétablissement.

CONCLUSIONS : À l'établissement des auteurs, la douleur associée à l'insertion de l'aiguille de biopsie pulmonaire est bien contrôlée. La douleur positionnelle est courante pour les patients qui doivent être en décubitus ventral. Parmi les solutions, soulignons mieux faire connaître la douleur positionnelle et adopter du matériel de soutien supplémentaire.

pain, need for medication and procedure duration. We anticipate that identifying and addressing patients' expectations, preferences and concerns will assist us in providing optimal pain management and comprehensive patient care.

\section{METHODS}

Coaxial fine-needle aspiration is the standard technique for computed tomography (CT)-guided transthoracic lung biopsies at the authors' institution. On average, three to five tissue samples are collected using a 20-gauge aspiration needle within a 19-gauge outer stabilizing needle. Patients receive $10 \mathrm{~mL}$ of $1 \%$ local lidocaine, $50 \mu \mathrm{g}$ of intravenous fentanyl and $1 \mathrm{mg}$ of intravenous midazolam before biopsy needle insertion to alleviate pain and anxiety. The duration of the procedure typically ranges from $60 \mathrm{~min}$ to $90 \mathrm{~min}$.

From March through June 2012, patients were contacted sequentially via telephone within one week of their procedure. The purpose of limiting data collection to seven days postprocedure was to maximize the accuracy and completeness of patients' recollections. A shorter

Joint Department of Medical Imaging, Toronto General Hospital, University of Toronto, Toronto, Ontario

Correspondence: Dr Demetris Patsios, Mount Sinai Hospital, New Clinical Services Building, Fifth Floor, Room 575, 600 University Avenue,

Toronto, Ontario M5G 1X5. Telephone 416-586-4800 ext 5598, e-mail demetris.patsios@uhn.ca 


\section{TABLE 1}

\section{Results of telephone calls to lung biopsy patients}

\begin{tabular}{lc}
\hline Total number of patients called & 49 \\
Unable to contact (long distance telephone number) & 9 \\
Unable to contact (no answer) & 15 \\
Total number of patients contacted & 25 \\
Excluded (not fluent in English) & 8 \\
Excluded (declined to participate) & 1 \\
Consented to participate & 16 \\
Age, years, mean \pm SD & $66.6 \pm 12.1$ \\
Age, years, range & $45-91$ \\
Interval following lung biopsy, days, mean \pm SD & $4.6 \pm 2.6$ \\
\hline
\end{tabular}

Data presented as $n$ unless otherwise indicated

\section{TABLE 2}

Characteristics of the study participants

\begin{tabular}{lccc}
\hline $\begin{array}{l}\text { Study } \\
\text { identification }\end{array}$ & Age, years & Sex & Position \\
\hline RK01 & 60 & Male & Prone \\
PW02 & 82 & Female & Prone \\
RP03 & 57 & Male & Prone \\
HP04 & 56 & Female & Supine \\
LS05 & 85 & Female & Prone \\
LH06 & 62 & Female & Supine \\
BE07 & 64 & Female & Prone \\
RM08 & 57 & Male & Prone \\
JL09* & 75 & Female & Prone \\
PA10 & 62 & Male & Prone \\
JL11* & 75 & Female & Prone \\
LB12 & 64 & Male & Supine \\
EC13 & 63 & Male & Supine \\
RR14 & 91 & Female & Prone \\
CC15 & 45 & Female & Supine \\
RJ16 & 67 & Female & Prone \\
\hline
\end{tabular}

*JL09 and JL11 represent one patient who had two independent lung biopsies

interval was not feasible due to the limited availability of study investigators. Telephone interviews were deemed to be the most efficient method for collecting semistructured qualitative data because they enable multiple patients to be contacted within a short period of time.

If patients did not answer their telephone after three calls, no further attempts were made. Voicemails were not left. Patients were excluded if they were $<18$ years of age, they were not fluent in English, they were enrolled in an interventional study with an investigational device or drug, or informed consent was not given. The study was explained in full at the outset of each telephone call to obtain verbal informed consent. If given, semistructured telephone interviews were conducted based on a study-specific interview template (Appendix 1). This was designed to assess patients' pain, informational needs and satisfaction. Open-ended questions were used to encourage study participants to elaborate on topics they deemed to be important (eg, "What aspects of your lung biopsy experience could have been improved? How so?"). Probing follow-up questions were used to clarify responses and elicit more detailed information. Responses were recorded on printouts of the interview template. Audio recordings were not obtained because of resource contraints. The interviews were approximately $15 \mathrm{~min}$ in duration. Data collection ceased at the point of data saturation $(n=16)$ as determined by one study author (EW). After completing 16 interviews, it was apparent that no new themes would materialize and additional data collection would be repetitive.

One author (EW) interviewed all study participants. The individual was not present for any of the lung biopsies. Participants were interviewed an average of 4.6 days after lung biopsy. The mean age of the patients was 66.6 years (range 45 years to 90 years) (Table 1). Six
TABLE 3

Qualitative themes

\begin{tabular}{ll}
\hline Lung biopsy pain & Procedural: "I didn't even know" \\
& Positional: "It's not terribly comfortable" \\
Patient education & If I had more information... \\
& What was unexpected \\
& No big surprises \\
\hline
\end{tabular}

men and 10 women participated. During their lung biopsy, 11 patients were prone and five patients were supine (Table 2). No patients withdrew from the study.

\section{Ethics}

The Research Ethics Board of Toronto General Hospital approved the study.

\section{Data analysis}

Qualitative study data were analyzed by one study author (EW) using thematic analysis, as outlined by Boyatzis (6). The coding phase consisted of identifying interview topics that were relevant to the study objectives. This generated a list of codes that was used to categorize interview data. The entire data set was re-examined throughout the study to ensure that emerging patterns were recognized. Related codes were combined into themes that accurately reflected the subject matter. Each theme was then analyzed with respect to the overall data set and study objectives. Illustrative excerpts were selected for theme naming and inclusion in the results section.

\section{RESULTS}

Attempts were made to contact 49 outpatients via telephone in the week following their lung biopsy. A total of 25 (51.0\%) patients were successfully reached and invited to participate in the study (Table 1). Of the contacted patients, $16(64.0 \%)$ met study criteria and constituted a consecutive sample.

The analysis yielded five themes that were divided into two primary categories: lung biopsy pain and patient education (Table 3). Pain was subdivided into procedural and positional pain. Procedural pain was defined as pain caused by insertion of the lung biopsy needle. Positional pain was defined as pain caused by positioning within the CT scanner. The three patient education themes pertained to informational needs, informational deficits and physician-patient communication.

\section{Lung biopsy pain}

Procedural pain: "I didn't even know": None of the study participants cited procedural pain as a problem. One patient expressed that "there was no pain whatsoever" and described the sensation as "more pressure than pain." Another explained feeling "about as much pain from the [biopsy] needle insertion as from the freezing [needle].” Intuitively, this represents an acceptable amount of pain for a lung biopsy. Perhaps the most convincing indication that procedural pain was well controlled was when one interviewee stated, "I didn't feel anything. I didn't even know it was done." In addition, all study participants indicated that they were satisfied with the amount of analgesic received.

Positional pain: "It's not terribly comfortable": Several patients experienced pain as a result of awkward positioning. Shoulder and neck pain were the most common concerns. Patients reported experiencing "shoulder pain because of the way [they were] positioned" and "[having] a bad stiff neck afterwards" due to the extensive time they were required to keep their head turned within the CT scanner, typically $60 \mathrm{~min}$ to $90 \mathrm{~min}$. The mean age of the study population was 66.6 years. This demographic may be more susceptible to positional pain due to an increased prevalence of comorbidities associated with older age such as osteoarthritis.

When it is feasible, patients' input about their preferred position was sought. One patient disclosed that they were "given a choice [to be 
prone or supine]" when asked about their intraprocedural positioning. However, in many cases, unnatural positioning is unavoidable because of the location of the lesion. Although standard hospital pillows are able to alleviate this problem in some patients, others believed that additional supportive equipment would be beneficial. One participant stated:

They should have some smaller pillows for when they elevate [patients' heads]

A notable observation is that all patients who reported positional pain underwent the procedure in the prone position. This suggests that careful attention should be devoted to ensure the comfort of this subset of patients.

\section{Patient education}

If I had more information...: The topic of informational needs surfaced during interviews when discussing patients' knowledge of the lung biopsy process. It was the first exposure to an interventional procedure for most of the participants, and anxiety was not uncommon. Providing additional patient education is one approach to minimizing anxiety, and was clearly valued by patients. One patient stated:

If I had more information of the process and how I would feel after and so on, that would have been helpful.

Requiring a lung biopsy can also result in a perceived loss of control, according to another interviewee:

I suppose because I knew nothing about it, my way of controlling was to gain some knowledge.

This is further evidence that patient education can be empowering. What was unexpected: Many of the comments regarding specific informational deficits were concerns about potential postprocedural complications. One interviewee was completely unaware of the most common complication - pneumothorax (4\% to $43 \%)(7-9)$ - and found it distressing to learn about it immediately before her procedure in the Medical Imaging Day Unit (MIDU):

What was unexpected was when I was lying [in the MIDU], [the radiologist] came and said to me that 'you could get a collapsed lung,' and I wasn't aware of that before I went in.

The potential for prolonged somnolence from midazolam surprised another participant. When asked about what was unexpected, she replied:

I guess the sedation [was unexpected], and actually I felt very tired for a couple of days afterwards which I didn't expect, kind of dopey.

Misunderstandings surrounding postprocedural diet and bathroom privileges also produced considerable angst in some patients.

No big surprises: Although patient education can be improved in certain areas, the theme of strong physician-patient communication emerged. One patient explained that "the doctor told [him] exactly what she was going to do, and did it." It was clear that the efforts made by radiologists to provide patients with comprehensive information were appreciated:

I was comfortable with [the radiologist] and he explained everything very clearly. He went through the procedure and told me what was going to happen. In the room [he said], 'Don't be surprised if you hear voices behind you. It will be the team.' There were no big surprises or not knowing what was going on.

\section{DISCUSSION}

The use of qualitative data was appropriate given the subjective nature of pain. A major advantage is that it can capture detailed, comprehensive information that may elude quantitative studies. In an unpublished questionnaire survey involving 47 lung biopsy patients conducted by our institution, it was found that $21.3 \%$ experienced no pain, $40.4 \%$ experienced mild pain, $17.0 \%$ experienced moderate pain and $21.3 \%$ experienced severe pain. The relatively large proportion of patients with moderate to severe pain raised questions about the adequacy of analgesia and sedation provided; it was unknown whether patients were satisfied with the current combination of medications they are given.

The results of the present study indicate that patients perceive minimal procedural pain. This implies that patients are receiving adequate analgesia before lung biopsy needle insertion. Conversely, the accounts of interviewed patients suggest that positional pain control may be suboptimal, resulting in substantial pain at times. On occasion, this pain was noted to persist well after lung biopsy completion, suggesting that it may occur despite appropriate analgesia. It is likely that this uncomfortable positioning was responsible for the severe pain reported on the initial questionnaire, which did not differentiate procedural from positional pain.

The position of the patient within the CT scanner varies on a caseby-case basis, depending on the safest and most efficient route of access to the mass or nodule of interest. As a result, patients may find themselves positioned uncomfortably for a prolonged period of time. One potential solution may be to increase the variety of cushions and supports available. Increasing awareness among the health care team is another intervention that may reduce positional pain; careful attention to patient comfort at the outset of procedures is likely to minimize residual postprocedural pain. Being cognizant of positional pain may also encourage radiologists to shorten procedure duration when possible. Together, these measures may help address what was previously an unrecognized problem.

The interview data signify that patient education can be improved, despite reports of high patient satisfaction. The two main informational deficiencies relate to potential complications and the postprocedural MIDU recovery period. Meeting these informational needs can likely be accomplished by further emphasizing complications and postprocedural restrictions to patients before their procedure through physician-patient communication. Dissemination of up-to-date patient education tools should also serve to inform patients and result in more accurate patient expectations.

To our knowledge, the present study is the first to qualitatively explore pain associated with CT-guided lung biopsies. Our literature search yielded many publications investigating diagnostic accuracy and complication rates, but pain management and patient education appear to be novel research topics. Similarly, there is a dearth of literature regarding pain management in liver and other CT-guided needle biopsies. Studies investigating ultrasound-guided biopsies are not useful comparisons because they often only require local analgesia, are shorter in duration and have less demanding positional requirements.

The primary limitation of the present study was the exclusive use of telephone interviews. Although they effectively captured dialogue, they were not sensitive to patients' facial expressions, body language or mood. Live interviews were not feasible because of the lung biopsy schedule, study duration and time limitations of the investigators. Another limitation was the inability to obtain audio recordings because of resource contraints. As a result, a minor amount of content was likely lost; however, manual transcription was effective in capturing the bulk of the subject matter.

A potential weakness of the study design is the exclusion of patients that do not speak English fluently. Pain management and patient education are likely more complicated in this population because of language barriers. Another possible selection bias may have been introduced by not being able to contact certain patients. Almost one-half of patients ( 24 of 49 ) were unable to be reached by telephone because they had a long-distance telephone number or did not answer (Table 1). The former may have skewed the study population toward a more urban demographic. Furthermore, patients who are difficult to contact via telephone may have reduced access to patient education resources. Despite these limitations, successfully contacted patients were eager to share their experiences, as evidenced by the $94.1 \%$ enrollment rate; 16 of the 17 contacted patients who met the 
inclusion criteria consented to participate (Table 1). The noninvasive nature of the present study likely contributed to this.

It can be concluded from the present study that pain associated with the insertion of lung biopsy needles is well controlled at our institution. The pain that is most significant to patients is associated with positioning within the CT scanner, particularly among those required to be in the prone position. In addition to increasing awareness of positional pain, we recommended instituting additional supportive equipment. The final conclusion that can be drawn is that patients desire and require more information, specifically concerning what to expect once the procedural aspect of their lung biopsy is complete. Fortunately, an excellent tool exists in the form of the University Health Network Lung Biopsy Patient Education Pamphlet (10). Increased distribution and future updates that include details of positional requirements, potential complications and MIDU recovery may serve to increase its utility.

Further research is necessary to determine whether these recommendations are sufficient to quantifiably reduce positional pain. Ultimately, the present study may pave the way for future research aimed at similar, minimally invasive procedures.

DISCLOSURES: The authors have no conflicts of interest to declare.

\footnotetext{
APPENDIX 1: INTERVIEW TEMPLATE

Qualitative assessment of pain management in patients undergoing lung biopsy

Study \#

Patient Information

Age:

Gender: M F Telephone\#:

Date of Interview:

Date of Lung Biopsy:

Section I: Pain history

1. Were you experiencing pain on admission? Where? What level of pain, on a scale of 0 to 10 ?

2. What was the cause(s) of this pain?

3. Have you been taking medication for pain on a regular basis before your lung biopsy?

4. What pain medication(s) were you taking? How much? How long?
}

\section{REFERENCES}

1. Watkinson AF, Francis IS, Torrie P, et al. Commentary: The role of anaesthesia in interventional radiology. Br J Radiol 2002;75:105-6.

2. Martin ML, Lennox PH. Sedation and analgesia in the interventional radiology department. J Vasc Interv Radiol 2003;14:1119-28.

3. England A, Tam CL, Thacker DE, et al. Patterns, incidence and predictive factors for pain after interventional radiology. Clin Radiol 2005;60:1188-94.

4. Mueller PR, Biswal S, Halpern EF, et al. Interventional radiologic procedures: Patient anxiety, perception of pain, understanding of procedure, and satisfaction with medication - a prospective study. Radiology 2000;215:684-8.

5. Schupp CJ, Berbaum K, Berbaum M, et al. Pain and anxiety during interventional radiologic procedures: Effect of patients' state anxiety at baseline and modulation by nonpharmacologic analgesia adjuncts. J Vasc Interv Radiol 2005;16:1585-92.

6. Boyatzis R. Transforming Qualitative Information: Thematic Analysis and Code Development. Thousand Oaks: Sage Publications, 1998.

\section{Section II: Pain management}

1. Were you lying face up or face down during your lung biopsy?

2. On a scale of 0 to 10 , with 0 representing no pain and 10 representing the worst possible pain, what number best indicates the maximum level of pain you experienced during your lung biopsy?

3. Was this pain caused by your lung biopsy?

4. Did you want pain medication during your lung biopsy? If not, why?

5. Did the health care team offer you pain medication during your lung biopsy? Did you accept? If not, why?

6. Did you ask for pain medication during your lung biopsy? If not, why?

7. Were you satisfied with the amount of time it took to receive pain medication after asking?

8. Were you satisfied with the amount of pain medication you received during your lung biopsy?

9. Were you comfortable during your lung biopsy? If not, why?

Section III: Lung biopsy experience

1. On a scale of 0 to 10 , with 0 representing no anxiety and 10 representing the worst possible anxiety, what number best indicates the level of anxiety you experienced on admission?

2. Were you sedated during your lung biopsy? If not, why?

3. Were you satisfied with the amount of sedation you received during your lung biopsy?

4. What aspects of your lung biopsy experience were unexpected? How could you have been better prepared?

5. Did you receive the lung biopsy patient education pamphlet? Was it helpful? If not, why?

6. Were you satisfied with the health care team's efforts to manage your pain? If not, why?

7. What aspects of your lung biopsy experience could have been improved? How so?

8. Do you have any additional comments regarding your lung biopsy experience?

7. Larscheid RC, Thorpe PE, Scott WJ. Percutaneous transthoracic needle aspiration biopsy: A comprehensive review of its current role in the diagnosis and treatment of lung tumors. Chest 1998;114:704-9.

8. Laspas F, Roussakis A, Efthimiadou R, et al. Percutaneous CT-guided fine-needle aspiration of pulmonary lesions: Results and complications in 409 patients. J Med Imaging Radiat Oncol 2008;52:458-62.

9. Wiener RS, Schwartz RM, Woloshin S, et al. Population-based risk for complications after transthoracic needle lung biopsy of a pulmonary nodule: An analysis of discharge records. Ann Intern Med 2011;155:137-44.

10. University Health Network. Lung biopsy: Information for patients. <www.uhn.ca/docs/HealthInfo/Shared\%20Documents/Lung_Biopsy. pdf> (Accessed July 26, 2012). 


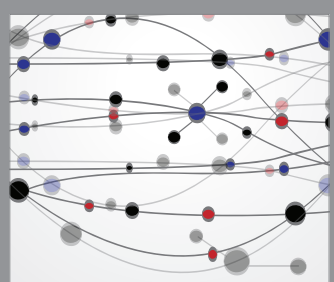

The Scientific World Journal
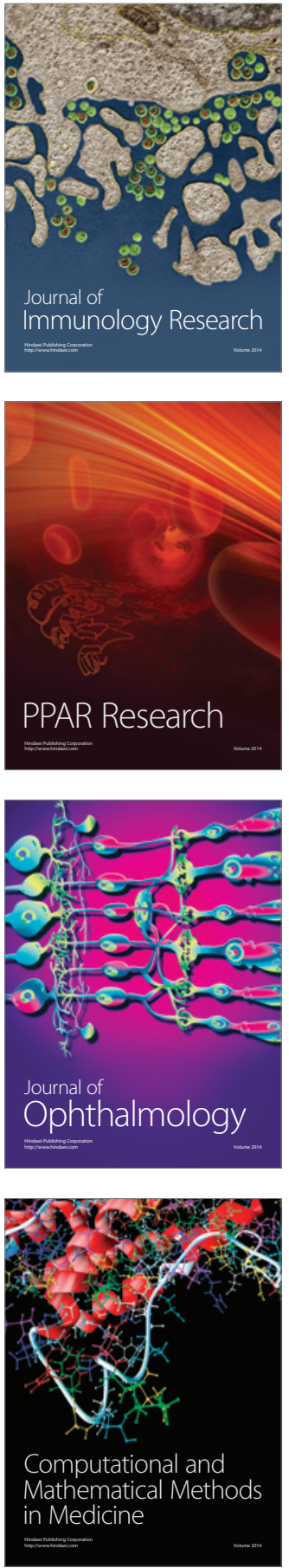

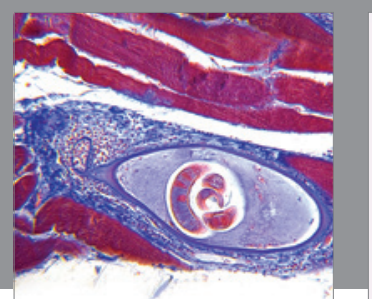

Gastroenterology Research and Practice

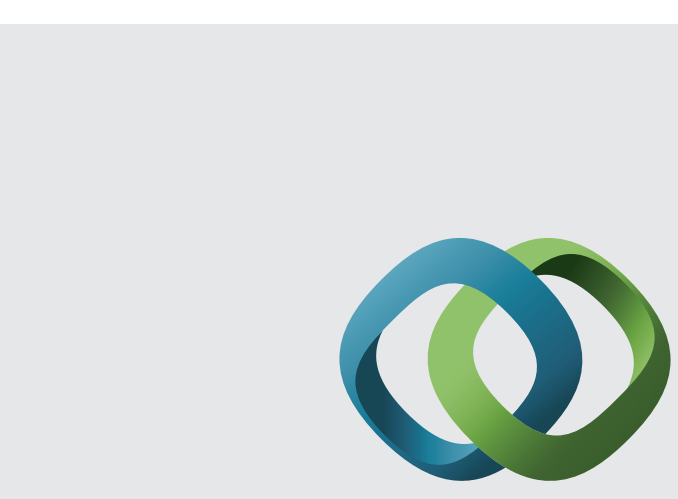

\section{Hindawi}

Submit your manuscripts at

http://www.hindawi.com
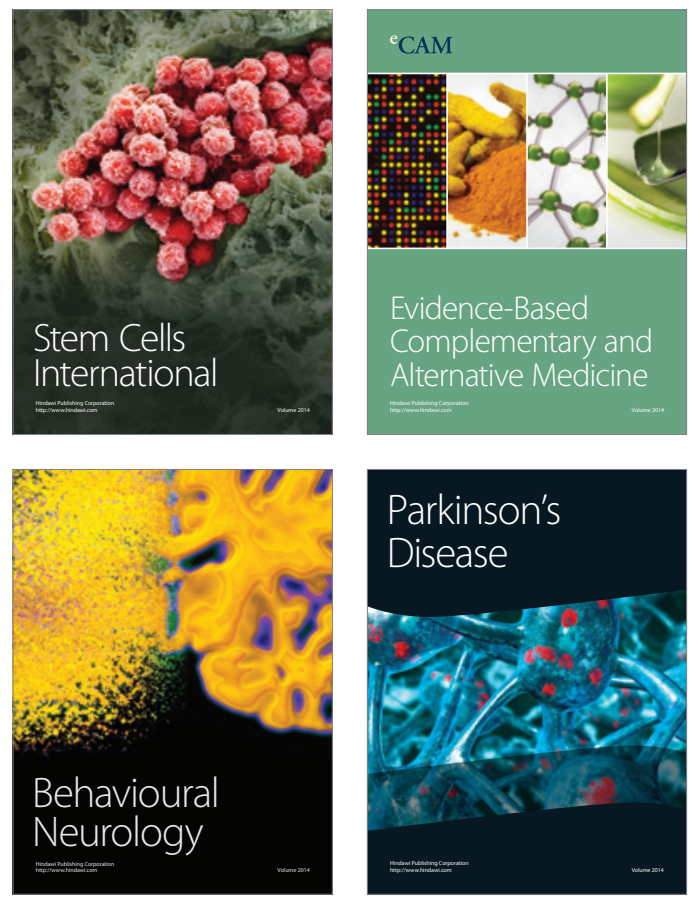
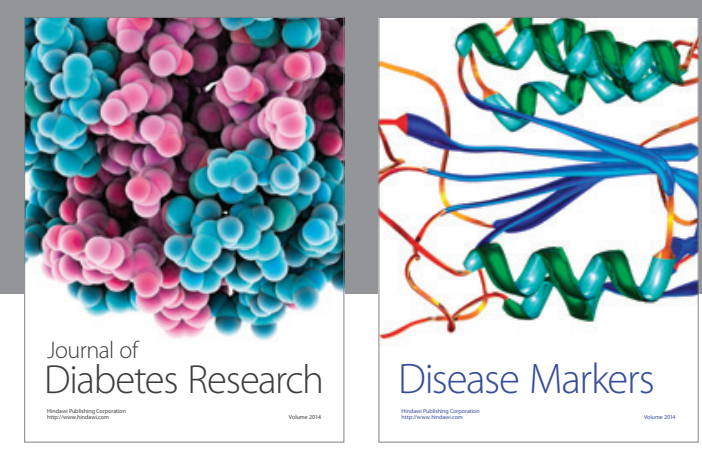

Disease Markers
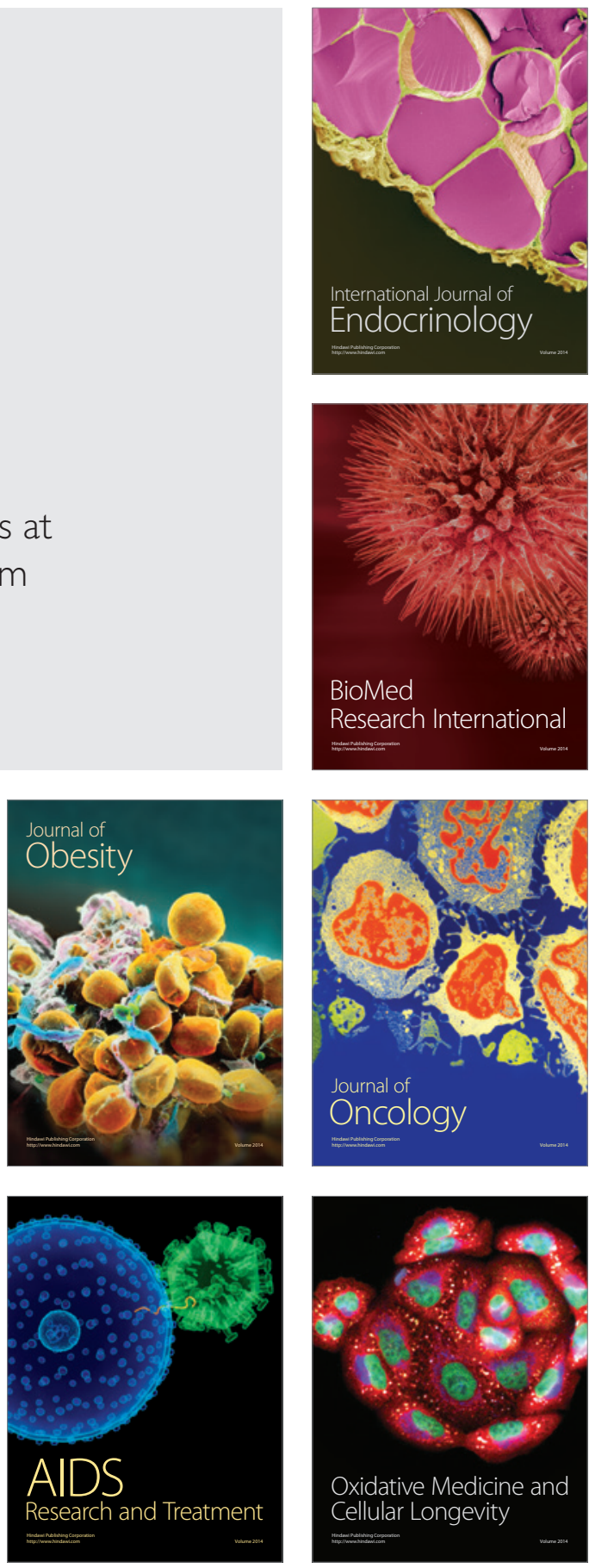